\title{
Effects of magnification and zooming on depth perception in digital stereomammography: an observer performance study
}

\author{
Heang-Ping Chan, Mitchell M Goodsitt, Lubomir M Hadjiiski, \\ Janet E Bailey, Katherine Klein, Katie L Darner and \\ Berkman Sahiner
}

Department of Radiology, University of Michigan, Ann Arbor, MI 48109, USA

E-mail: chanhp@umich.edu

Received 15 May 2003

Published 4 November 2003

Online at stacks.iop.org/PMB/48/3721

\begin{abstract}
We are evaluating the application of stereoscopic imaging to digital mammography. In the current study, we investigated the effects of magnification and zooming on depth perception. A modular phantom was designed which contained six layers of 1-mm-thick Lexan plates, each spaced $1 \mathrm{~mm}$ apart. Eight to nine small, thin nylon fibrils were pasted on each plate in horizontal or vertical orientations such that they formed 25 crossing fibril pairs in a projected image. The depth separation between each fibril pair ranged from 2 to $10 \mathrm{~mm}$. A change in the order of the Lexan plates changed the depth separation of the two fibrils in a pair. Stereoscopic image pairs of the phantom were acquired with a GE full-field digital mammography system. Three different phantom configurations were imaged. All images were obtained using a $\mathrm{Rh}$ target/Rh filter spectrum at $30 \mathrm{kVp}$ tube potential and $\mathrm{a} \pm 3^{\circ}$ stereo shift angle. Images were acquired in both contact and $1.8 \mathrm{X}$ magnification geometry and an exposure range of 4 to $63 \mathrm{mAs}$ was employed. The images were displayed on a Barco monitor driven by a Metheus stereo graphics board and viewed with LCD stereo glasses. Five observers participated in the study. Each observer visually judged whether the vertical fibril was in front of or behind the horizontal fibril in each fibril pair. It was found that the accuracy of depth discrimination increased with increasing fibril depth separation and x-ray exposure. The accuracy was not improved by electronic display zooming of the contact stereo images by $2 \mathrm{X}$. Under conditions of high noise (low mAs) and small depth separation between the fibrils, the observers' depth discrimination ability was significantly better in stereo images acquired with geometric magnification than in images acquired with a contact technique and displayed with or without zooming. Under our experimental conditions, a $2 \mathrm{~mm}$ depth discrimination was achieved with over $60 \%$ accuracy on contact images with and without zooming, and with over
\end{abstract}


$90 \%$ accuracy on magnification images. This study indicates that stereoscopic imaging, especially with magnification, may be useful for visualizing the spatial distribution of microcalcifications in a cluster and for differentiating overlapping tissues from masses on mammograms.

\section{Introduction}

At present, x-ray mammography is the only diagnostic procedure with a proven capability for detecting early stage, clinically occult breast cancers (Seidman et al 1987). Although mammography has a high sensitivity for detection of breast cancers when compared to other diagnostic modalities, studies indicate that radiologists do not detect all carcinomas that are visible on retrospective analyses of the images (Wallis et al 1991, Bird et al 1992, Harvey et al 1993, Beam et al 1996). These missed detections are often a result of the very subtle nature of the radiographic findings. However, one of the major deficiencies of mammography is its inability to discern lesions hidden behind dense fibroglandular tissues (Jackson et al 1993). It is estimated that about $20 \%$ of the breast cancers in dense breasts are not detected by mammography (Wallis et al 1991, Bird et al 1992). It is therefore important to improve the sensitivity of mammography in imaging dense breasts. With the advent of high resolution digital detectors, new breast imaging techniques such as stereomammography (Goodsitt et al 2000, 2002, Chan et al 2000, 2001, 2002a, 2002b, Getty et al 2001, Maidment et al 2003), digital tomosynthesis (Niklason et al 1997, Suryanarayanan et al 2000) and computed tomography (Raptopoulos et al 1996, Boone et al 2001) are being developed to alleviate this problem.

A conventional radiograph is a projection image. The anatomical structures along the $\mathrm{x}$-ray beam path are projected onto a two-dimensional image plane and overlap with each other. The overlying tissue structures often obscure the visibility of subtle lesions of interest in a radiograph. The camouflaging effect of the anatomical structures is the main cause of missed diagnosis. Stereoscopic imaging will allow the overlying structures to be perceived at different depths, thereby reducing the camouflaging effect. It has been reported that digital stereomammography allowed the detection of additional lesions that were obscured on screenfilm mammograms (Getty et al 2001).

Stereoscopic radiography has been attempted for different types of examinations (Doi et al 1981, Kelsey et al 1982, Doi and Duda 1983, Higashida et al 1988, Trocme et al 1990, Ragnarsson and Karrholm 1992). The principle of stereoscopic imaging is shown in figure 1. The $\mathrm{x}$-ray focal spot is shifted, along a direction parallel to the image plane, to the left and the right of the central axis to obtain two images of the object. The object has to remain stationary during the process. The images are referred to as the left-eye (LE) and the right-eye (RE) images. When the two images are positioned properly and viewed by trained eyes or with the aid of a stereoscope so that the left eye sees only the LE image and the right eye sees only the RE image, the parallax between the two images creates the depth perception. It is important to match the amount of stereoscopic shift to the imaging geometry to obtain best depth perception with minimal eyestrain. In general, a larger stereoscopic shift produces improved depth perception; however, as the shift increases, it becomes more difficult for the observer to fuse the images for the stereoscopic effect, and eye fatigue increases. According to Christensen's Physics of Diagnostic Radiology (Curry et al 1992), early radiologists determined empirically that a tube shift of $10 \%$ of the source-to-film distance worked well. This translates to a stereo shift angle of about $\pm 3^{\circ}\left(\tan ^{-1}(0.05) \cong 3^{\circ}\right)$ relative to a normal to the detector. 

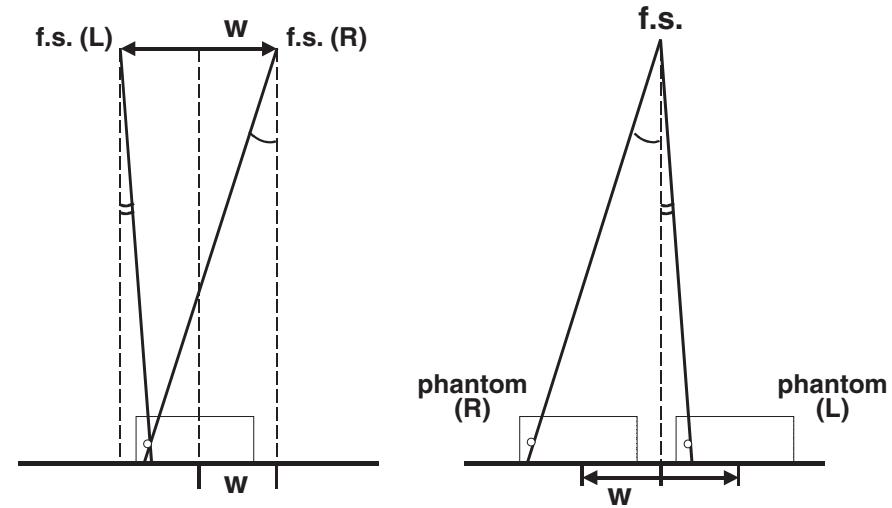

Figure 1. Imaging geometry for acquisition of stereoscopic image pairs. On the left is a conventional 'focal-spot-shift' method in which the focal spot is shifted to the left and to the right by a distance of $w$ to expose the left-eye and right-eye images. On the right is an equivalent 'phantom-shift' method in which the phantom is shifted to the left and the right of the central ray by a distance of $w$. The 'phantom (R)' geometry corresponds to the 'f.s. (R)' geometry, and the 'phantom (L)' geometry corresponds to the 'f.s. (L)' geometry.

Stereoscopic imaging has not achieved widespread acceptance in clinical practice, mainly because of the doubled film cost and increased patient exposure (Curry et al 1992). A secondary problem is the need to train the eyes to perceive the stereoscopic effect without aid, or to use a special stereoscope, with careful arrangement of the films. Digital imaging may make the stereoscopic technique a viable approach because no additional film cost will be required. Furthermore, a digital detector has a wider linear-response range and a higher contrast sensitivity than a screen-film system so that good-quality images may be acquired at a reduced radiation dose. Images in digital form can be subjected to image processing, further enhancing the visibility of the image details. Digital stereoscopic images can be viewed on a single electronic display device that displays the left-eye and right-eye images alternately at a high refresh rate. The observer views the displayed images through a stereo goggle such as a pair of liquid crystal display (LCD) glasses. The LCD panels act as electronic shutters, blocking the light to the left eye and the right eye alternately. When the goggle is synchronized with the display so that only the left eye can see the left-eye image and only the right eye can see the right-eye image in rapid succession, the parallax between the two images will create a stereo effect. The stereoscopic images can be viewed singly in the conventional manner or stereoscopically on the same viewing station to provide complementary diagnostic information.

An additional advantage of stereoscopic imaging is the three-dimensional (3D) information it provides on the lesions of interest. It has been reported that the 3D distribution of microcalcifications may be correlated with the malignant or benign nature of the cluster (Conant et al 1996, Maidment et al 1996). Spiculations from a mass may be more readily distinguished from overlapping tissues under stereoscopic viewing conditions. This supplementary diagnostic information may improve the classification of malignant and benign lesions, thereby reducing unnecessary biopsies and increasing the positive predictive value of mammography.

We are evaluating the application of stereoscopic techniques to digital mammography. Previously we studied the effects of stereo shift and imaging conditions on the depth perception of fibrils in stereo phantom images (Chan et al 2000, 2001). We also demonstrated that a virtual cursor could provide accurate depth measurements in stereo phantom images acquired 


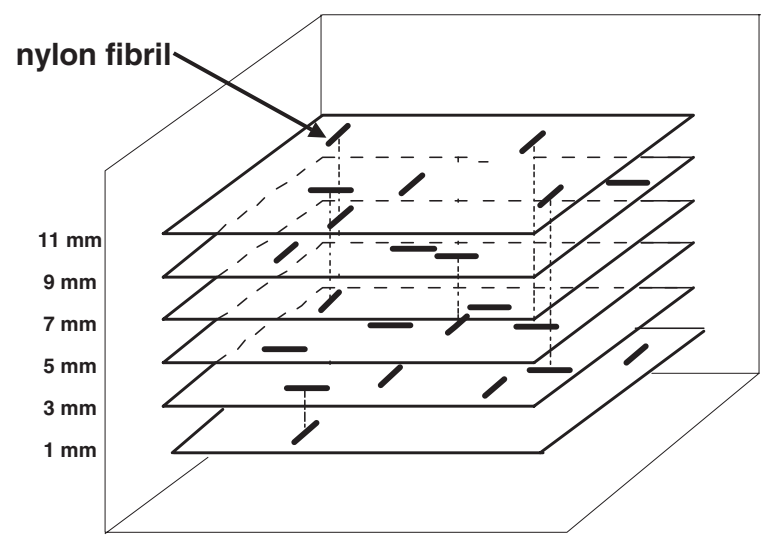

Figure 2. A modular stereo phantom consists of six layers of Lexan plates on which nylon fibrils were pasted either in the horizontal or the vertical direction in one of the $5 \times 5$ matrix locations on one of the plates. By properly arranging 50 fibrils on the six plates, the projected image will contain 25 pairs of crossing fibrils with different depth separations between the two fibrils.

under mammographic conditions (Goodsitt et al 2000, 2002). In the present study, we further evaluated the effects of geometric magnification, display zooming and $\mathrm{x}$-ray exposure on the visual depth discrimination of fibril-like objects in stereo phantom mammograms.

\section{Materials and methods}

\subsection{Modular stereo phantom}

We have designed a modular stereo phantom for evaluation of depth perception in stereomammography. A schematic of the phantom is shown in figure 2. The phantom consists of six 1-mm-thick Lexan sheets, each separated by 1-mm-thick spacers. Each Lexan plate contains a $5 \times 5$ array of object areas. Fifty nylon fibrils, each about $8 \mathrm{~mm}$ in length and $0.53 \mathrm{~mm}$ in diameter, are arranged in these object areas. Twenty-five fibrils are oriented perpendicular and another 25 are oriented parallel to the stereo shift direction. Henceforth, we will refer to the fibrils oriented in the perpendicular direction as 'vertical' and the fibrils oriented in the parallel direction as 'horizontal'. On average, eight to nine fibrils are placed in the object areas on each Lexan plate. The location and orientation of a fibril on a Lexan plate are randomly chosen with the constraint that, in the projection image, each object area contains the projection of one vertical and one horizontal fibril that cross each other. These plates can be arranged in different orders to produce many independent object configurations, i.e., the depth separation and whether the vertical fibril is in front of or behind the horizontal fibril in a given pair change when the order of the plates changes. Different types of test objects such as microcalcifications may be used in place of the fibrils to generate a different phantom although only fibrils were used in the present study. An additional 1-mm-thick Lexan plate without objects is placed on top of the phantom to protect the test objects on the top layer. The total thickness of Lexan in the phantom is therefore $7 \mathrm{~mm}$.

\subsection{Image acquisition and display}

Digital stereoscopic image pairs were acquired with a GE Senographe 2000D digital mammography system. The system employs a digital detector consisting of a CsI:Tl scintillator and an amorphous-Si active matrix flat panel. The detector measures $23 \mathrm{~cm} \times 18 \mathrm{~cm}$, with 


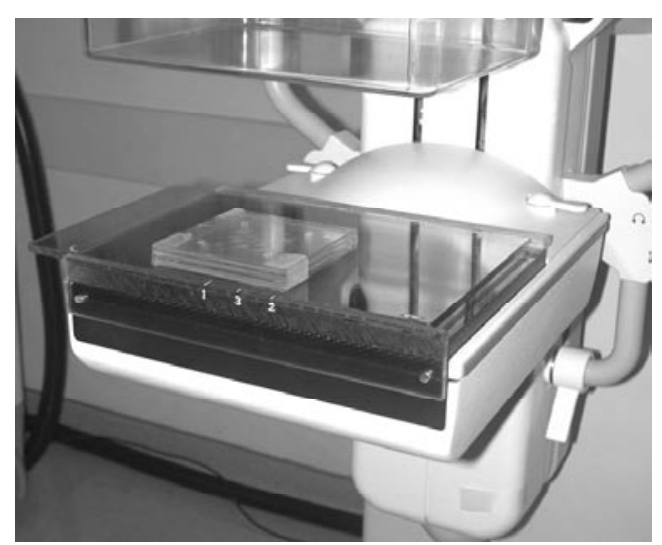

(a)

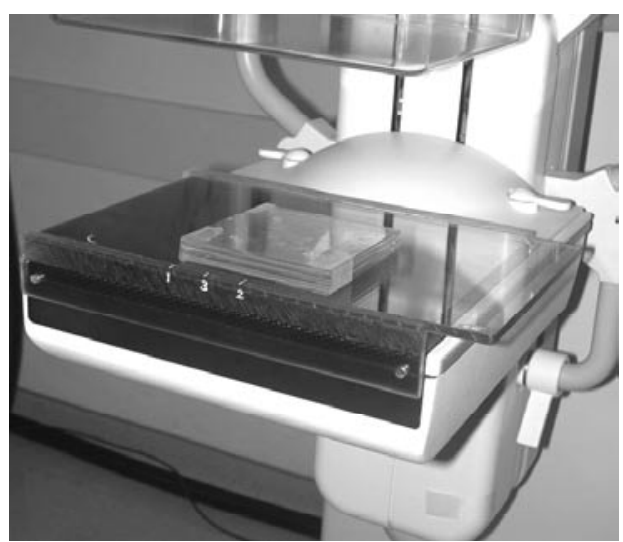

(b)

Figure 3. The phantom platform placed on top of the detector of the digital mammography system in the setup for contact imaging. The sliding plate with the stereo phantom was shifted to the left in (a) and to the right in (b) at a distance equivalent to $\mathrm{a} \pm 3^{\circ}$ stereo angle.

a pixel size of $100 \mu \mathrm{m} \times 100 \mu \mathrm{m}$. We acquired stereoscopic pairs of images using x-ray techniques of $30 \mathrm{kVp}, \mathrm{Rh}$ target/Rh filter, a $\pm 3^{\circ}$ stereo angle, contact (reciprocating grid, $0.3 \mathrm{~mm}$ focal spot) and $1.8 \mathrm{X}$ magnification (no grid, $0.15 \mathrm{~mm}$ focal spot) geometries and exposures of 4, 8, 32 and $63 \mathrm{mAs}$ per image. A relatively hard x-ray beam was used to produce lower contrast images, thus making the perception task more challenging.

Since the digital mammography system was not designed for stereo imaging, it is not easy to move the $\mathrm{x}$-ray focal spot to the angulated positions at $\pm 3^{\circ}$ from the central ray for imaging the stereo pair. We used an equivalent imaging geometry (figure 1) in which the phantom was shifted instead of the x-ray source. Comparing the two geometries in figure 1, it can be seen that the position of the $\mathrm{x}$-ray source relative to the phantom is the same except that the focal-spot-to-detector distance is slightly shorter in the phantom-shift geometry because the $\mathrm{x}$-ray source moves along an arc. However, with the $3^{\circ}$ shift about a fulcrum of rotation at a distance of $46 \mathrm{~cm}$ from the $\mathrm{x}$-ray focal spot, this error is less than $0.1 \%$. Using the geometry of the x-ray system, we calculated that a $\pm 3^{\circ}$ stereo shift of the $\mathrm{x}$-ray focal spot is equivalent to a phantom shift distance of $\pm 2.4 \mathrm{~cm}$ from its central position. The shift distance is the same for either the contact geometry or the magnification geometry.

We built a phantom platform with Lexan (figure 3) to move the phantom from one position to the other in a direction parallel to the chest wall for acquisition of the stereo pair. The platform has a stationary base that is fitted on the Bucky holder for contact geometry or on the magnification stand for magnification geometry. A sliding plate on top of the base is used to support and move the phantom parallel to the chest wall direction of the mammography system. The sliding plate is guided by two guardrails parallel to the chest wall to ensure a precise and reproducible movement. The total thickness of the Lexan platform is $1.7 \mathrm{~cm}$. During image acquisition, care was taken such that the phantom remained stationary on the sliding plate while the plate was moved between the two shifted positions. Pictures of the phantom platform at the left and right shifted positions in the contact geometry are shown in figure 3.

Three different configurations of the fibril phantoms were imaged under each exposure condition. Twenty-four $(=3$ phantoms $\times 4$ exposures $\times 2$ geometries $)$ stereo image pairs were thus produced. For each exposure and geometry condition, there were a total of 75 pairs of fibril images ( 25 fibril pairs in each phantom configuration $\times 3$ configurations) at 


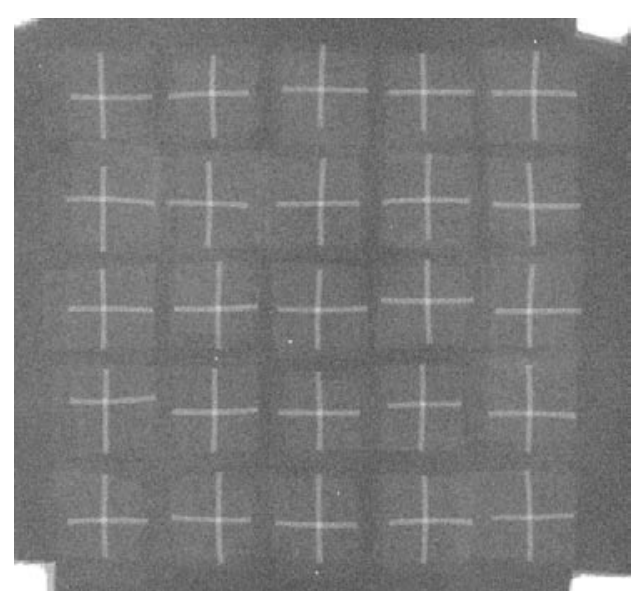

(a)

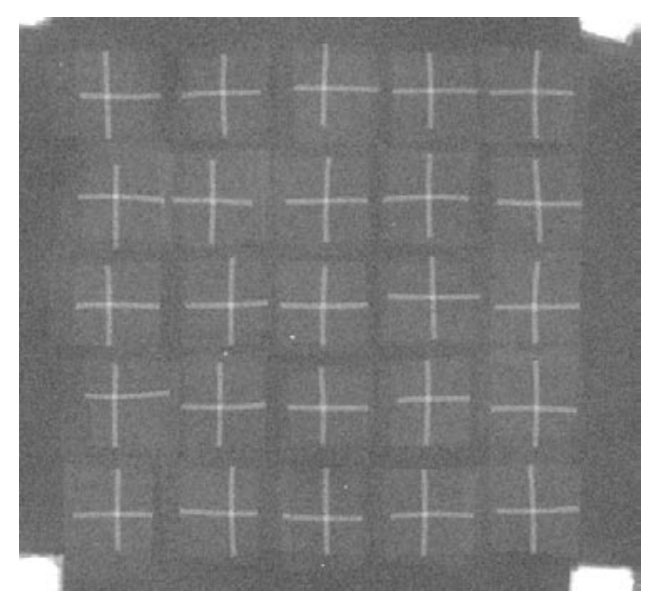

(b)

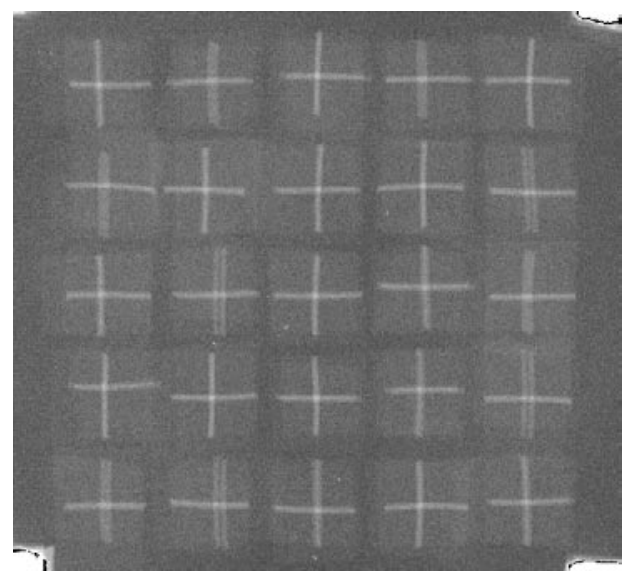

(c)

Figure 4. A stereo image pair acquired in contact geometry: (a) left-eye image, (b) right-eye image, (c) displayed stereo image - the 25 pairs of crossing fibrils showed different degrees of stereo shift due to the different depth separations between the two fibrils.

five different depth separations $(2,4,6,8,10 \mathrm{~mm})$ to be evaluated. An example of a stereo image pair acquired with contact geometry is shown in figure 4.

The images were displayed on a $21^{\prime \prime}(\sim 53 \mathrm{~cm})$ Barco-Metheus (Beaverton, OR) model 521 display monitor driven by a Barco-Metheus model 1760S stereoscopic board and a SUN Microsystems (Palo Alto, CA) Ultra 10 computer using in-house developed software. The Metheus board displays $1408 \times 1408 \times 8$ bit images at a refresh rate of $114 \mathrm{~Hz}$. It operates in a page flipping stereoscopic mode with the left- and right-eye images displayed alternately. NuVision (Beaverton, OR) LCD stereoscopic glasses were used for viewing the stereoscopic images. The stereo workstation is shown in figure 5.

\subsection{Observer experiment}

Five observers including two experienced mammographic radiologists participated in the experiment. All observers took a standard Randot Circles Stereo test (Stereo Optical Co., Inc., Chicago, IL) to evaluate their stereo acuity. In this test, the observers viewed ten rows of test 


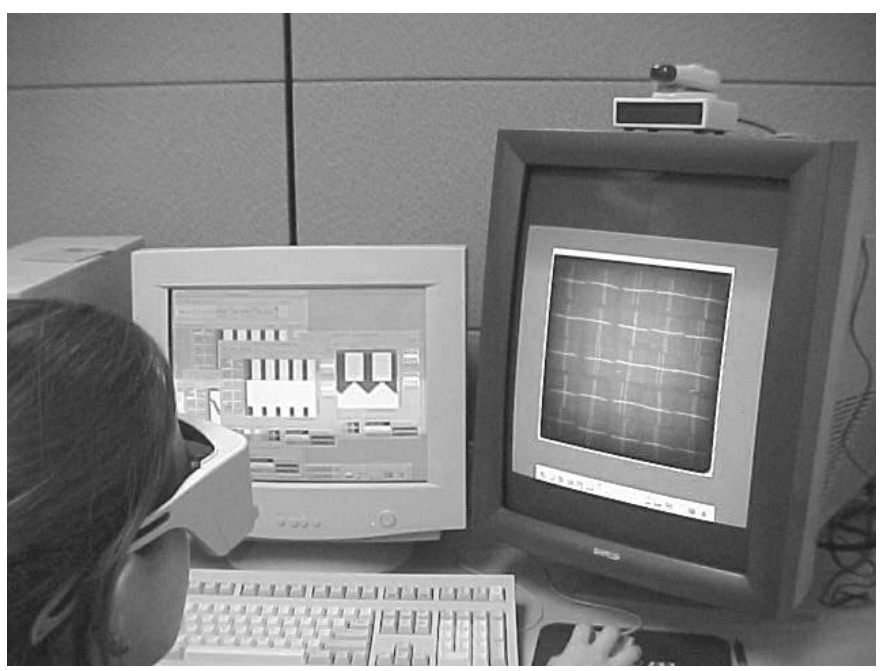

Figure 5. The SUN-based Barco-Metheus stereo display workstation used in the observer study.

objects through polarized glasses. Each row contains three objects, one of which is the object that exhibits the stereo effect and appears to be on a plane different from the other two objects. The difference progressively decreases due to decreasing stereo shift, and thus increasing the degree of difficulty. The observers had to determine which one was the stereo object in each row. All of our observers could correctly identify all the objects in the test, indicating that their stereo acuity was at least $20 \mathrm{~s}$ of arc at a viewing distance of $16^{\prime \prime}(\sim 40 \mathrm{~cm})$. Their performance is comparable to the average performance $(21.3 \mathrm{~s}$ of arc) for adults with excellent, balanced monocular visual acuity measured with this test pattern (Simons 1981).

For our experiment, the task for the observers was to visually judge whether the vertical fibril in each pair of overlapping fibrils was in front of (closer to the observer) or behind the horizontal fibril. The observer was not informed of the truth after reading each image. Each observer read the images sequentially in a different randomized order. The reading order of the fibril pairs in the $5 \times 5$ matrix in a given image was changed randomly by changing the starting location and the reading direction, e.g., from the upper left corner and by row, from the upper left corner and by column, from the lower right corner and by row, etc in an effort to further reduce any potential effects of memorization. The contact images were read in two modes - in a regular and in a $2 \mathrm{X}$-zoom mode, referred to as the contact and contactzoom mode, respectively. The $1.8 \mathrm{X}$ geometric magnification images were read only in the regular mode, referred to as the magnification mode. The contact and the contact-zoom modes of the same image were not read consecutively so that it was unlikely that the observer would remember the results of the other mode. The observers were not informed of the conditions under which the image being viewed was acquired, although the difference in image sizes between the contact and the magnification or contact-zoom images was apparent. Prior to reading the test cases, the observers participated in training sessions to become familiar with the reading task.

\section{Results}

In this study, we quantified the accuracy of depth perception in stereo images by an observer as the percentage of correct decisions to differentiate the relative depths of the fibrils. The 


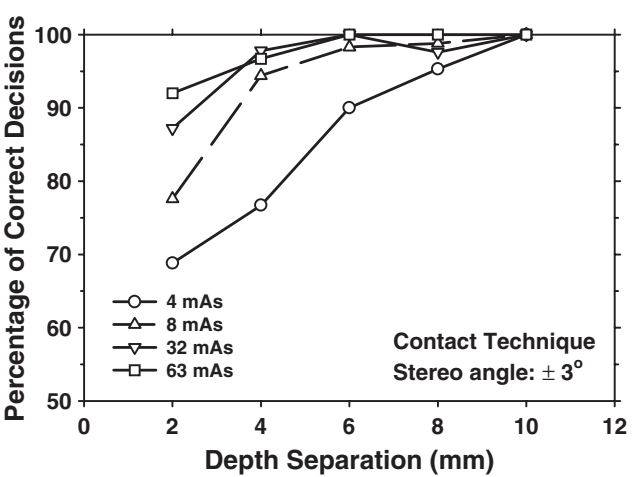

(a)

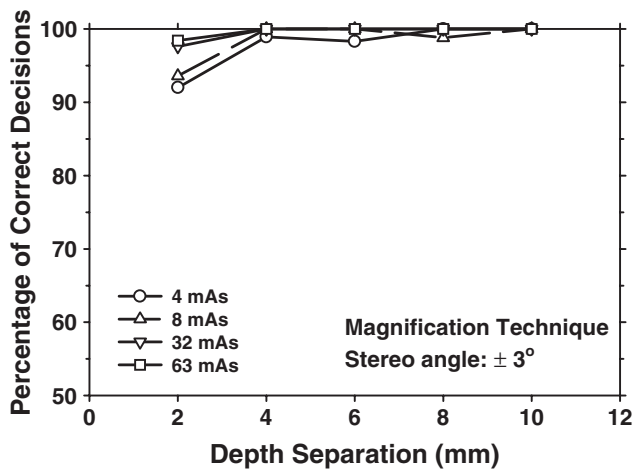

(c)

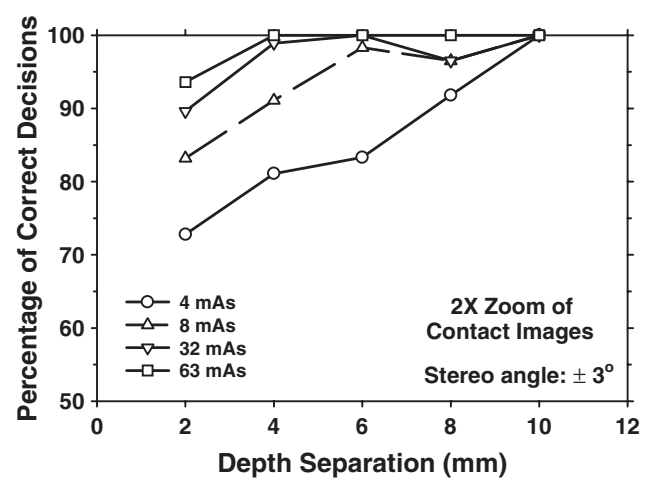

(b)

Figure 6. Percentage of correct decisions in differentiating the relative depths of two crossing fibrils, averaged over five observers. (a) Stereo images acquired with contact geometry, (b) stereo images acquired with contact geometry, displayed with $2 \mathrm{X}$ zoom and (c) stereo images acquired with $1.8 \mathrm{X}$ geometric magnification.

results were averaged over the five observers. The dependences of the average percentage of correct decisions on the depth separation of the crossing fibrils and the exposure levels for the contact, contact-zoom and magnification modes are shown in figures 6(a)-(c), respectively. Generally, the percentage of correct decisions increases as the exposure increases and as the depth separation between the two fibrils increases. The dependence on the depth separation diminishes as the exposure increases. At small depth separations, the noise in the images has a much stronger influence on the depth perception. The percentage of correct decisions increases rapidly as the exposure increases. At a depth separation of $10 \mathrm{~mm}$, the percentage of correct decisions was $100 \%$ for all observers in all three modes, regardless of exposure levels. The curves for contact mode are very similar to those for the contact-zoom mode. On the other hand, the curves for the magnification mode are much higher than those for the contact or contact-zoom mode. The standard deviation of the percentage of correct decisions varied from 0 to about $13 \%$ for the contact mode and the contact-zoom mode, and varied from 0 to $6.7 \%$ for the magnification mode. The standard deviation generally increased as the percentage of correct decisions decreased. The standard deviation was zero for the conditions in which the average percentage of correct decisions was $100 \%$ because all observers were $100 \%$ correct in their decisions.

The statistical significance of the differences between every two modes, contact versus contact-zoom, contact versus magnification and contact-zoom versus magnification, was 
Table 1. The $p$-values from the two-tailed paired $t$-test of the differences in the percentage of correct decisions in differentiating the depths of two crossing fibrils between the contact and the magnification modes. The entries indicated by '-' have $p$-values $>0.05$ so that the differences are not statistically significant. The two entries indicated by -* have $p$-values of 0.089 and 0.065 , close to being significant.

\begin{tabular}{lllll}
\hline \multirow{2}{*}{$\begin{array}{l}\text { Depth separation } \\
(\mathrm{mm})\end{array}$} & \multicolumn{5}{c}{ Exposure } \\
\cline { 2 - 5 } & $4 \mathrm{mAs}$ & $8 \mathrm{mAs}$ & $32 \mathrm{mAs}$ & $63 \mathrm{mAs}$ \\
\hline 2 & 0.007 & 0.000 & $-^{*}$ & - \\
4 & 0.009 & $-^{*}$ & - & - \\
6 & 0.034 & - & - & - \\
8 & - & - & - & - \\
10 & - & - & - & - \\
\hline
\end{tabular}

Table 2. The $p$-values from the two-tailed paired $t$-test of the differences in the percentage of correct decisions in differentiating the depths of two crossing fibrils between the contact-zoom and the magnification modes. The entries indicated by '-' have $p$-values $>0.05$ so that the differences are not statistically significant.

\begin{tabular}{lllll}
\hline \multirow{2}{*}{$\begin{array}{l}\text { Depth separation } \\
(\mathrm{mm})\end{array}$} & \multicolumn{5}{c}{ Exposure } \\
\cline { 2 - 5 } & $4 \mathrm{mAs}$ & $8 \mathrm{mAs}$ & $32 \mathrm{mAs}$ & $63 \mathrm{mAs}$ \\
\hline 2 & 0.014 & 0.003 & 0.011 & - \\
4 & 0.016 & 0.003 & - & - \\
6 & 0.021 & - & - & - \\
8 & - & - & - & - \\
10 & - & - & - & - \\
\hline
\end{tabular}

estimated by a two-tailed paired $t$-test. The paired $t$-test was performed for each depth separation and exposure level over the five observers. The $p$-values for the contact-versusmagnification and contact-zoom-versus-magnification for the various exposure and depth separation conditions are shown in tables 1 and 2, respectively. The differences between the contact and the magnification modes are statistically significant ( $p$-value $<0.05$ ) for depth separations of $2 \mathrm{~mm}$ to $6 \mathrm{~mm}$ at $4 \mathrm{mAs}$, and for a $2 \mathrm{~mm}$ separation at $8 \mathrm{mAs}$. The differences between the contact-zoom and the magnification modes are statistically significant for depth separations of $2 \mathrm{~mm}$ to $6 \mathrm{~mm}$ at $4 \mathrm{mAs}$, for $2 \mathrm{~mm}$ to $4 \mathrm{~mm}$ separations at $8 \mathrm{mAs}$ and for a $2 \mathrm{~mm}$ separation at $32 \mathrm{mAs}$. The differences between the contact and contact-zoom modes are not statistically significant.

We further analysed separately the reading results for two subgroups of crossing fibrils, one subgroup with the vertical fibril in front of (referred to as the 'front group') and the other behind (referred to as the 'back group') the horizontal fibril. The average percentage of correct decisions as a function of depth separation was obtained for each exposure level, similar to the analysis for the entire group described above. We observed that the average percentage of correct decisions for the front group was often greater than that for the back group for a given exposure and a given depth separation. A histogram of the differences between the average percentage of correct decisions for the front group and that for the back group under the corresponding conditions is plotted in figure 7 for the 60 conditions studied ( 4 exposure levels $\times$ 5 depth separations $\times 3$ modes). Of the 60 differences, only three were negative, i.e., the average percentage of correct decisions for the front group was smaller than that for the back group. All 28 zeroes (no difference) happened when both the front group and the back group were $100 \%$ correct. These occurred when the depth separations were large and the exposures 


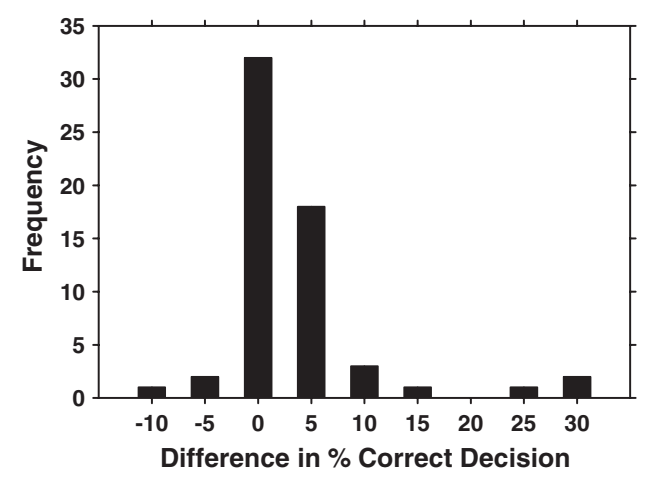

Figure 7. Histogram of the difference in the percentage of correct decisions between the front group (vertical fibril in front of the horizontal fibril) and the back group (vertical fibril behind the horizontal fibril).

were high. The other 29 differences were positive, i.e., the percentage of correct decisions for the front group was larger than that for the back group. However, paired $t$-tests of the differences between the front and the back groups under each condition did not reach statistical significance, probably due to the smaller numbers of samples when they were separated into subgroups.

\section{Discussion}

Depth perception in stereomammography depends on a number of factors including the x-ray exposure, depth separation and imaging geometry. In this study, we investigated the dependence of the percentage of correct decisions in differentiating the relative depths of two crossing fibrils on these factors using images acquired with $\pm 3^{\circ}$ stereo angle. We found that a $2 \mathrm{~mm}$ depth resolution could be achieved with over $60 \%$ accuracy for all imaging conditions studied. For contact geometry, the accuracy improved to greater than $90 \%$ at higher exposures. Magnification stereomammography provided over $90 \%$ accuracy at a $2 \mathrm{~mm}$ depth resolution for all exposure levels studied. An interesting finding was that displaying the stereo images acquired with contact geometry in $2 \mathrm{X}$-zoom mode did not improve the depth discrimination accuracy. In contrast, geometric magnification with about the same factor (1.8X) of enlargement significantly improved depth perception. This result indicated that the signal-to-noise ratio (SNR) might be a more important factor affecting depth perception than the perceived object size. When the images are acquired with geometric magnification, both the spatial resolution of the detector relative to the object and the X-ray quanta per unit object area increase (Doi and Rossmann 1974, Doi and Imhof 1977), although geometric unsharpness may somewhat reduce the gain in resolution. The improved detector resolution and reduced quantum mottle contributed to an increase in the SNR of the image. On the other hand, if the images were acquired in contact geometry and the object size was enlarged electronically by zooming during display, the inherent SNR of the object in the images remained fixed. The differences in the perceived SNRs (Loo et al 1985, Aufrichtig 1999) between the contact mode and the contact-zoom modes would be mainly caused by the change in the perceived noise of the display monitor relative to the object size as well as the change in the perceived signal and image noise spectra due to zooming relative to the frequency response of the observer's visual system. Display zooming may have a strong influence if the perception is limited by 


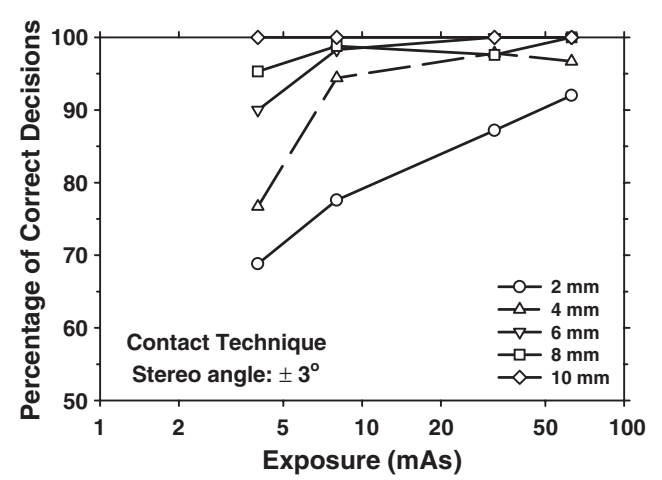

(a)

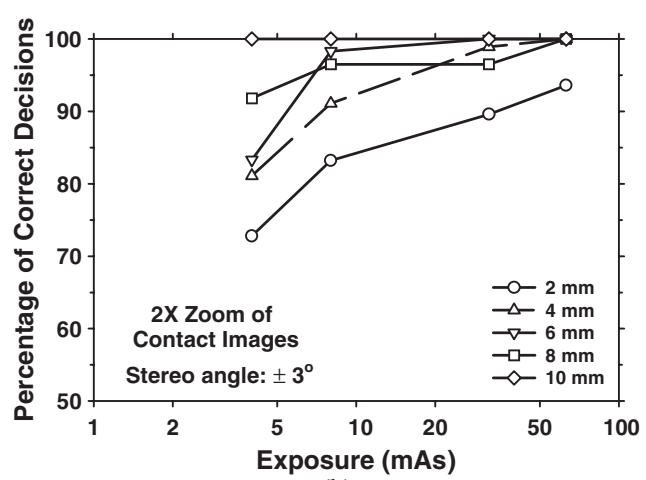

(b)

Figure 8. Dependence of the percentage of correction decisions on the logarithm of exposure. (a) Contact mode. (b) Contact-zoom mode.

the resolution of the human visual system. In our case, the objects were relatively large and it was unlikely that they were near the resolution limit of the observers' visual system. The improved SNR inherent in the geometrically magnified images may therefore have a dominant effect on depth discrimination compared with that of increased displayed object size. This may be the reason that display zoom did not have a strong impact on the accuracy of depth perception in this study.

We plotted the average percentage of correct decisions as a function of log exposure (mAs) for each depth separation between the two crossing fibrils in the pair as shown in figures 8(a) and (b), respectively, for the contact and contact-zoom modes. The average percentages of correct decisions for the magnification mode under all conditions were greater than $90 \%$ and were not plotted. Three of the data points at a $8 \mathrm{~mm}$ depth separation appear to be lower than those at a $6 \mathrm{~mm}$ depth separation for a given exposure. Since the standard deviation at each of these data points is about twice as large as the difference between the percentage of correct decisions at $6 \mathrm{~mm}$ and $8 \mathrm{~mm}$ separations, the apparent local reverse of the trend can likely be attributed to experimental uncertainties. From the curves of the contact and contactzoom modes, it appears that there is no simple relationship between the percentage of correct decisions and log exposure. The curve shape varied among the different depth separations and they were not linear. The percentage of correct decisions increased with exposure more rapidly at low dose levels and levelled off at high doses. Depth discrimination is different from a single-object detection task. The relationships among the perceived depth separation, the SNRs of the individual objects and the percentage of correct decisions are still unknown. Further studies are needed to explore how depth discrimination is related to the SNR of the individual objects and whether the depth discrimination task can be predicted by SNR models.

The intraobserver variability was estimated from two repeated readings of the set of test images by one observer. The difference between the two readings for each exposure level and depth separation was calculated. The difference in the percentage of correct decisions between the two readings varied from 0 to $20 \%$, from 0 to $8 \%$ and from 0 to $8 \%$, respectively, for the contact, contact-zoom and magnification modes. The variability generally increased with decreasing depth separation and decreasing exposure. There were no differences between the two readings at depth separations of $8 \mathrm{~mm}$ and $10 \mathrm{~mm}$ for all exposure levels and all image modes. For the magnification mode, the two repeated readings were identical for 18 of the 20 conditions ( 5 depth separations $\times 4$ exposure levels). A difference was observed only at a $2 \mathrm{~mm}$ separation with the two lower exposure levels, $4 \mathrm{mAs}$ and $8 \mathrm{mAs}$. For both the contact 
and contact-zoom modes, 14 of the 20 conditions were identical. Four of the six differences occurred at a $2 \mathrm{~mm}$ separation with the four exposure levels (4, 8, 32, $63 \mathrm{mAs})$.

In a previous study, we designed a virtual cursor to measure the absolute depths of the fibrils in stereo phantom images similar to those used in this study. We found that the accuracy of the absolute depth measurement also depended on the image modes. The accuracy of the measurements in contact-zoom mode was similar to those in contact mode, whereas the accuracy was higher when measured in the images acquired with geometric magnification (Goodsitt et al 2002). Although these results are consistent with the observation in the current depth perception experiments, the task of visual depth discrimination and the task of absolute depth measurement are different. The former task requires the differentiation of the relative depths of two objects, whereas, the latter task measures the absolute depth of a single object with a calibrated virtual cursor. We found that an observer who could perform the depth discrimination task very well might fail to make accurate depth measurements. In clinical practice, visual depth discrimination is likely to be the more important task during detection and diagnostic workup. Depth measurement is likely to be more important for treatment planning and intervention.

We used a thin phantom $(7 \mathrm{~mm})$ for the experiments in this study. A thin phantom would result in less scattered radiation than a thick phantom (e.g., 4 to $5 \mathrm{~cm}$ thick for an average breast). The effect of scattered radiation in digital imaging is mainly a reduction of the SNR for a given exposure. In this study, we reduced the SNR by reducing the exposure. The overall effect of increased scatter on the percentage of correct decision for a given exposure would be similar to that of lower x-ray exposure. Since scattered radiation is reduced by the air gap in the magnification geometry and by the Bucky grid in the contact geometry, the effect of scatter would be relatively small even if a thicker phantom was used. In our previous study (Chan et al 2000, 2001), we sandwiched the modular phantom between two $2 \mathrm{~cm}$ thick BR-12 breast tissue equivalent material to simulate the scatter of an average breast. The relative trend between different exposure conditions observed in the previous study was similar to that observed in the current study. We therefore expect that the relative trend of the different imaging conditions will not be affected by the thickness of the phantom.

In this study, acquisition of the stereo image pair was accomplished by shifting the phantom; there was no grid cutoff due to misalignment of the x-ray beam and the focused grid. In patient imaging, the stereo image pair has to be acquired by shifting the focal spot as shown in figure 1(a). The central ray of the x-ray beam will not be aligned with the central axis of the grid, thus artefacts due to grid cutoff may occur. We have performed a pilot study acquiring stereomammograms of a few patients with Institutional Review Board approval and patient informed consent. We did not observe obvious grid cutoff artefacts in the displayed stereomammograms, probably because the grid ratio is low and the stereo shift is small. If $3 \mathrm{D}$ image acquisition methods such as digital tomosynthesis and stereomammography are implemented clinically in the future, we believe that a new grid design should be developed for the digital mammography system so that there will not be a trade-off between SNR and $3 \mathrm{D}$ information when using these new imaging techniques.

In this study, we used test objects containing a crossing fibril pair perpendicular to each other with the horizontal fibril parallel to the stereo shift direction. We observed a trend that the depth discrimination was poorer if the horizontal fibril was in front of the crossing pair. In our previous study of absolute depth measurement with a virtual cursor (Goodsitt et al 2000), we also found that the accuracy of measuring the depth of horizontal fibrils was much worse than that of vertical fibrils. However, in actual clinical images, there will be few, if any, objects of interest that consist of linear and uniform structures exactly parallel to each other and parallel to the stereo shift direction. Therefore, the angular orientation of the two 
objects to be discriminated will not be a major issue in clinical practice as long as they contain structures that are oriented at a moderate angle relative to the stereo shift direction.

\section{Conclusions}

The accuracy of depth discrimination of fibrils in stereomammography increases with x-ray exposure and depth separation and depends on imaging geometry. At a stereo angle of $\pm 3^{\circ}$, a $2 \mathrm{~mm}$ depth resolution was achieved with over $60 \%$ accuracy for all imaging conditions studied. It improved to greater than $90 \%$ accuracy at higher doses in contact geometry. With magnification stereomammography, a $2 \mathrm{~mm}$ depth resolution was achieved with greater than $90 \%$ accuracy for all exposure levels studied. It was found that zooming the contact stereo images by $2 \mathrm{X}$ did not improve the accuracy under our experimental conditions. When the images were noisy and the depth separation between the fibrils was small, depth discrimination was significantly better in stereo images acquired with geometric magnification than in images acquired with a contact technique and displayed with or without zooming. These results indicate that stereoscopic imaging, and in particular, magnification stereomammography, may be useful for visualizing the spatial distribution of microcalcifications in a cluster and differentiating overlapping tissues from masses on mammograms. Further studies are underway to investigate the dependence of depth perception on the shape and size of the objects and to evaluate if specially designed cursors can assist in depth discrimination of target objects in stereoscopic images. An observer experiment is being conducted to evaluate the characterization of mammographic lesions in stereo images of biopsy tissue specimens (Chan et al 2003).

\section{Acknowledgments}

This work is supported by US Army Medical Research and Materiel Command Grants DAMD 17-98-1-8210 and DAMD17-99-1-9294. The content of this publication does not necessarily reflect the position of the funding agency, and no official endorsement of any equipment and product of any companies mentioned in this publication should be inferred.

\section{References}

Aufrichtig R 1999 Comparison of low contrast detectability between a digital amorphous silicon and a screen-film based imaging system for thoracic radiography Med. Phys. 26 1349-58

Beam C A, Layde P M and Sullivan D C 1996 Variability in the interpretation of screening mammograms by US radiologists-findings from a national sample Arch. Intern. Med. 156 209-13

Bird R E, Wallace T W and Yankaskas B C 1992 Analysis of cancers missed at screening mammography Radiology 184 613-7

Boone J M, Nelson T R, Lindfors K K and Seibert J A 2001 Dedicated breast CT: radiation dose and image quality evaluation Radiology $221657-67$

Chan H P, Goodsitt M M, Darner K L, Sullivan J M, Hadjiiski L M, Petrick N and Sahiner B 2001 Effects of stereoscopic imaging technique on depth discrimination Proc. IWDM 2000—5th Int. Workshop on Digital Mammography, Digital Mammography (Toronto, Canada) ed M J Yaffe (Madison, WI: Medical Physics Publishing) pp 13-8

Chan H P, Goodsitt M M, Hadjiiski L, Bailey J E, Klein K, Darner K L and Paramagul C 2002a Digital stereomammography: observer performance study of the effects of magnification and zooming on depth perception Proc. SPIE 4682 163-6

Chan H P, Goodsitt M M, Hadjiiski L M, Helvie M A, Bailey J, Klein K and Roubidoux M A 2002b Development of digital stereo imaging technique for mammography Era of Hope Meeting (U.S. Army Medical Research and Material Command, Department of Defense, Breast Cancer Research Program, Orlando, Florida, September 25-28) 
Chan H P, Goodsitt M M, Hadjiiski L, Roubidoux M A, Bailey J E, Helvie M A, Lydick J T and Sahiner B 2003 ROC study comparing radiologists' performances in evaluating breast lesions on stereoscopic and single-projection digital specimen mammograms Med. Phys. 301456 (abstract)

Chan H P, Goodsitt M M, Sullivan J M, Darner K L and Hadjiiski L M 2000 Depth perception in digital stereoscopic mammography Era of Hope Meeting (U.S. Army Medical Research and Material Command, Department of Defense, Breast Cancer Research Program, Atlanta, Georgia, June 8-12)

Conant E F, Maidment A D, Albert M, Piccoli C W, Nussbaum S A and McCue P A 1996 Small field-of-view digital imaging of breast calcifications: method to improve diagnostic specificity Radiology 201(P) 369

Curry T S, Dowdey J E and Murry R C 1992 Christensen's Physics of Diagnostic Radiology 4th edn (Philadelphia, PA: Lea and Febiger)

Doi K and Duda E E 1983 Detectability of depth information by use of magnification stereoscopic technique in cerebral angiography Radiology 146 91-5

Doi K and Imhof H 1977 Noise reduction by radiographic magnification Radiology 122 479-87

Doi K, Patronas N J, Duda E E, Geldner E and Dietz K 1981 X-ray imaging of blood vessels to the brain by use of magnification stereoscopic technique Adv. Neurol. 30 175-89

Doi K and Rossmann K 1974 The effect of radiographic magnification on blood vessel imaging with various screenfilm systems Med. Phys. $1257-61$

Getty D J, Pickett R M and D’Orsi C J 2001 Stereoscopic digital mammography: improving detection and diagnosis of breast cancer Computer Assisted Radiology and Surgery 2001, Proc. 15th Int. Congress and Exhibition (Berlin) (International Congress Series 1230) ed H U Lemke, M W Vannier, K Inamura, A G Farman and K Doi (Amsterdam: Elsevier) pp 506-11

Goodsitt M M, Chan H P, Darner K L and Hadjiiski L M 2002 The effects of stereo shift angle, geometric magnification and display zoom on depth measurements in digital stereomammography Med. Phys. 29 2725-34

Goodsitt M M, Chan H P and Hadjiiski L M 2000 Stereomammography: evaluation of depth perception using a virtual 3D cursor Med. Phys. 27 1305-10

Harvey J A, Fajardo L L and Innis C A 1993 Previous mammograms in patients with impalpable breast carcinomas: retrospective vs blinded interpretation Am. J. Roentgenol. 161 1167-72

Higashida Y, Hirata Y, Saito R, Doudanuki S, Bussaka H and Takahashi M 1988 Depth determination on stereoscopic digital subtraction angiograms Radiology $168560-2$

Jackson V P, Hendrick R E, Feig S A and Kopans D B 1993 Imaging of the radiographically dense breast Radiology 188 297-301

Kelsey C A, Moseley R D, Mettler S A and Briscoe D E 1982 Cost-effectiveness of stereoscopic radiographs in detection of lung nodules Radiology 142 611-3

Loo L N, Doi K and Metz C E 1985 Investigation of basic imaging properties in digital radiography: 4. Effect of unsharp masking on the detectability of simple patterns Med. Phys. 12 209-14

Maidment A D A, Albert M, Conant E F, Piccoli C W and McCue P A 1996 Prototype workstation for 3-D diagnosis of breast calcifications Radiology 201(P) 556

Maidment A D A, Bakic P and Alberg M 2003 Is stereomammography possible without increasing dose? Proc. IWDM 2002_6th Int. Workshop on Digital Mammography, Digital Mammography (Bremen, Germany) ed H Petitgen (Berlin: Springer) pp 510-5

Niklason L T et al 1997 Digital tomosynthesis in breast imaging Radiology 205 399-406

Ragnarsson J I and Karrholm J 1992 Factors influencing postoperative movement in displaced femoral neck fractures: evaluation by conventional radiography and stereoradiography J. Orthop. Trauma 6 152-8

Raptopoulos V, Baum J K, Hochman M, Karellas A, Houlihan M J and D’Orsi C J 1996 High resolution CT mammography of surgical biopsy specimens J. Comput. Assist. Tomogr. 20 179-84

Seidman H, Gelb S K, Silverberg E, LaVerda N and Lubera J A 1987 Survival experience in the breast cancer detection demonstration project CA Cancer J. Clin. 37 258-90

Simons K 1981 Stereoacuity norms in young children Arch. Ophthalmol. 99 435-45

Suryanarayanan S, Karellas A, Vedantham S, Glick S J, D’Orsi C J, Baker S P and Webber R L 2000 Comparison of tomosynthesis methods used with digital mammography Acad. Radiol. 7 1085-97

Trocme M C, Sather A H and An K N 1990 A biplanar cephalometric stereoradiography technique Am. J. Orthod. Dentofacial Orthop. 98 168-75

Wallis M G, Walsh M T and Lee J R 1991 A review of false negative mammography in a symptomatic population Clin. Radiol. 44 13-5 